\title{
Prediction of Large for Gestational Age by Ultrasound at 35 Weeks and Impact of Ultrasound-Delivery Interval: Comparison of 6 Standards
}

\author{
Ricardo Savirón-Cornudella ${ }^{\mathrm{a}}$ Luis M. Esteban ${ }^{\mathrm{b}}$ Rocío Aznar-Gimeno ${ }^{c}$ \\ Peña Dieste Pérez ${ }^{d}$ Faustino R. Pérez-López ${ }^{e}$ Berta Castán-Larraz ${ }^{f}$ \\ Gerardo Sanz $^{g}$ Mauricio Tajada-Duaso ${ }^{d}$
}

\begin{abstract}
aDepartment of Obstetrics and Gynecology, Villalba General Hospital, Madrid, Spain; ${ }^{b}$ Escuela Universitaria Politécnica de La Almunia, Universidad de Zaragoza, Zaragoza, Spain; 'Instituto Tecnológico de Aragon, ITAINNOVA,

Zaragoza, Spain; ${ }^{d}$ Department of Obstetrics and Gynecology, Miguel Servet University Hospital, Zaragoza, Spain;

eDepartment of Obstetrics and Gynecology, University of Zaragoza Faculty of Medicine and Instituto de Investigaciones

Sanitarias Aragón, Zaragoza, Spain; 'Department of Obstetrics and Gynecology, San Pedro Hospital, Logroño, Spain;

9Department of Statistical Methods and Institute for Biocomputation and Physics of Complex Systems-BIFI,

University of Zaragoza, Zaragoza, Spain
\end{abstract}

\section{Keywords}

Adverse perinatal outcomes · Large for gestational age ·

Birth weight · Estimated fetal weight · Estimated percentile weight · Fetal growth standard · INTERGROWTH-21st ·

Ultrasound $\cdot$ WHO fetal growth charts

\begin{abstract}
Objective: The aim of the study was to assess the predictive ability of the ultrasound estimated percentile weight (EPW) at 35 weeks to predict large for gestational age (LGA) at term delivery according to 6 growth standards, including population, population-customized, and international references. The secondary objectives were to determine its predictive ability to detect adverse perinatal outcomes (APOs) and whether the ultrasound-delivery interval influences the detection rate of LGA newborns. Methods: This was a retrospective cohort study of 9,585 singleton pregnancies. Maternal clinical characteristics, fetal ultrasound data obtained at 35 weeks, and pregnancy and perinatal outcomes were used
\end{abstract}

to calculate EPWs to predict LGAs at delivery according to the customized and the non-customized (NC) Miguel Servet University Hospital (MSUH), the customized Figueras, the NC Fetal Medicine Foundation (FMF), the NC INTERGROWTH$21 \mathrm{st}$, and the NC World Health Organization (WHO) standards. Results: For a 10\% false-positive rate, detection rates for total LGAs at delivery ranged from $31.2 \%$ with the WHO (area under the curve [AUC] 0.77; 95\% confidence interval $[\mathrm{CI}], 0.76-0.79$ ) to $56.5 \%$ with the FMF standard (AUC 0.85; $95 \% \mathrm{Cl}, 0.84-0.86)$. Detection rates and values of AUCs to predict LGAs by ultrasound-delivery interval (range 1-6 weeks) show higher detection rates as the interval decreases. APO detection rates ranged from $2.5 \%$ with the WHO to $12.6 \%$ with the Figueras standard. Conclusion: The predictive ability of ultrasound estimated fetal weight at 35 weeks to detect LGA infants is significantly greater for FMF and MSUH NC standards. In contrast, the APO detection rate is significantly greater for customized standards. The shorter ultrasound-delivery interval relates to better prediction rates. karger@karger.com

www.karger.com/fdt

Karger (c) 2020 S. Karger AG, Base
Ricardo Savirón-Cornudella

Department of Obstetrics and Gynecology, Hospital General de Villalba Calle Alpedrete 41

ES-28400 Collado Villalba (Spain)

rsaviron@gmail.com 


\section{Introduction}

Screening for fetal growth abnormalities is an essential component of antenatal care, and fetal ultrasound plays a key role in this issue. Greater perinatal morbidity has been observed in large for gestational age (LGA) newborns [1] and stillbirth [2]. ISUOG Practice Guidelines for ultrasound assessment of fetal growth consider LGA fetuses as those with an estimated fetal weight (EFW) above the 90th centile, although the 95th centile, 97th centile, +2 standard deviations, and $Z$-score deviation have also been referred as cutoffs in the literature [3]. A randomized controlled trial published in 2015 showed that labor induction may decrease perinatal morbidity in newborns weighing more than $4,000 \mathrm{~g}$ or above the 95 th percentile [4], thus the clinical interest in the early detection of LGAs. In addition, as has been suggested, universal versus selective LGA screening in the 3 rd trimester improves the detection rate at delivery [5].

Since ultrasound assessment allows professionals to monitor intrauterine growth, different fetal growth standards have been based on the EFW to calculate the estimated percentile weight (EPW) using Hadlock et al.'s [6, 7] methodology or more recent studies that apply multilevel models. Although studies such as the one conducted by Gardosi et al. [8] have claimed that these standards can also be customized to maternal and fetal physiological variables, other studies have questioned the validity of customized standards $[9,10]$. Moreover, international population standards have recently been published by EFW [11], including those from the INTERGROWTH21 st project $[12,13]$ and the World Health Organization (WHO) [14].

In order to provide new knowledge on the performance of growth standards, the main objective of this study was to compare 6 growth standards, customized and non-customized (NC), local and international ones, to detect LGA in term newborns by ultrasound at 35 weeks. With the same purpose, the secondary objective was to determine the predictive ability to detect adverse perinatal outcomes (APOs) and whether the ultrasound-delivery interval influences the detection rate of LGA newborns.

\section{Methods}

\section{Study Design}

This was a retrospective cohort study of births assisted at the Miguel Servet University Hospital (MSUH) between March 2012 and December 2016. The inclusion criteria were as follows: (1) live singleton pregnancies controlled in MSUH from the 1st trimester

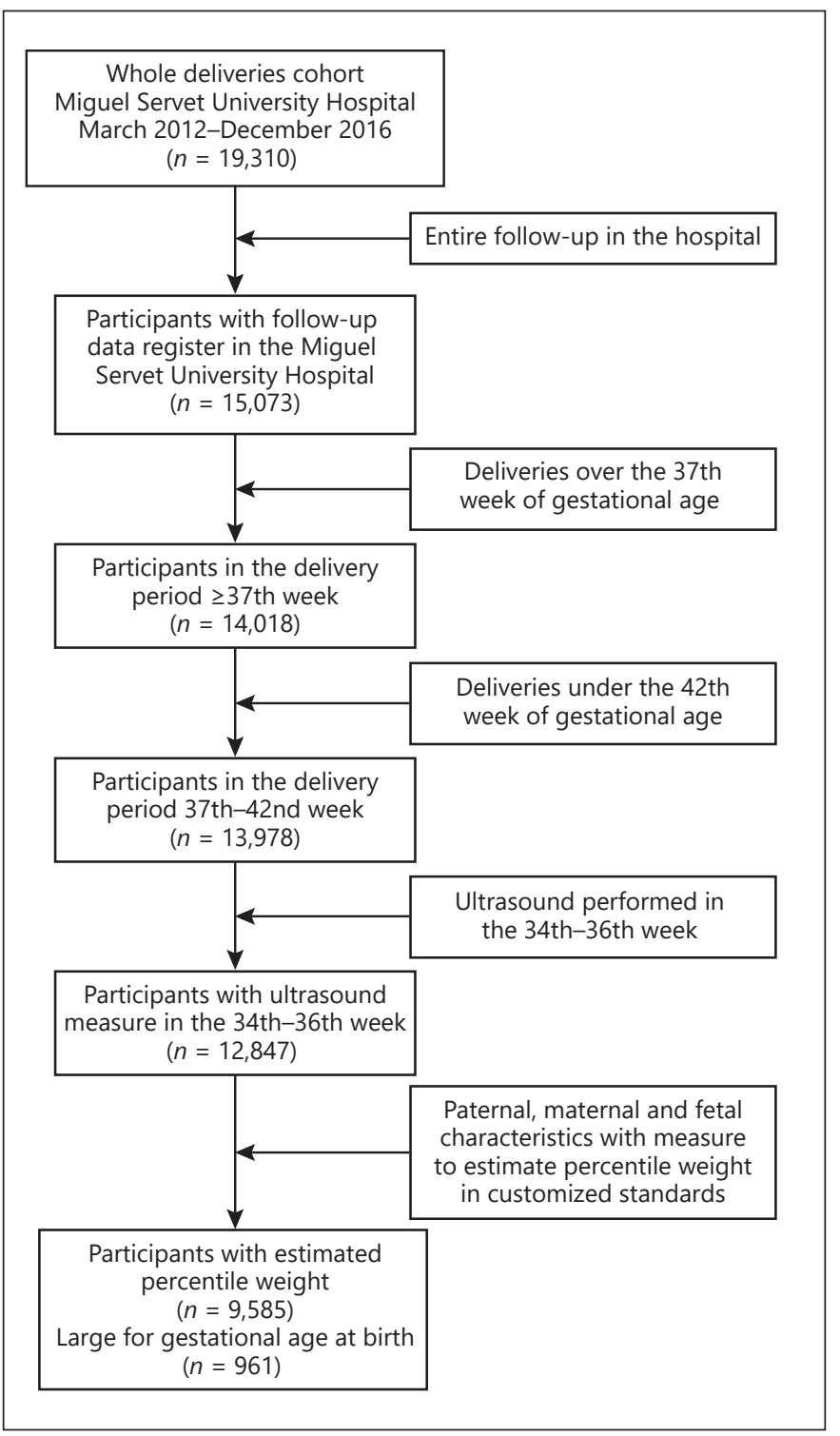

Fig. 1. Flowchart of patient recruitment.

of gestation, (2) fetal ultrasound assessment at a gestational age of 35 (range 34-36) weeks, and (3) deliveries between 37 and 42 weeks of gestational age with fetuses without stillbirth associated with malformations or chromosomal abnormalities. From the 19,310 consecutive deliveries assisted in our hospital in the period studied, the 9,585 cases which fulfilled the specific inclusion criteria such as data availability to estimate percentile weights by standards were considered for the analysis. Selection of study participants is detailed in Figure 1. Our research was approved by the Clinical Research Ethics Committee of Aragon (PI20/414).

The last menstrual period was adjusted by means of 1 st trimester ultrasound results [15]. Universal ultrasound screening was performed at 35 weeks (range 34-36 weeks) at the Ultrasound and Prenatal Diagnosis Unit using either Voluson 730 Expert, E6, E8 (General Electric Healthcare, Zipf, Austria) or Aloka ProSound 
Table 1. Parental baseline characteristics (top), and pregnancy (middle) and perinatal characteristics (bottom) of pregnancies

\begin{tabular}{|c|c|}
\hline Clinical characteristic & Pregnancies $(n=9,585)$ \\
\hline \multicolumn{2}{|l|}{ Parental characteristic } \\
\hline Maternal age, years & $33.3(30.1-36.1)$ \\
\hline Maternal BMI, $\mathrm{kg} / \mathrm{m}^{2}$ & $23.2(21.1-26.2)$ \\
\hline Maternal height, $\mathrm{cm}$ & $163(159-168)$ \\
\hline Paternal height, $\mathrm{cm}$ & $176(172-181)$ \\
\hline \multicolumn{2}{|l|}{ Parity } \\
\hline 0 & $5,077(53.0 \%)$ \\
\hline 1 & $3,724(38.9 \%)$ \\
\hline$\geq 2$ & $784(8.1 \%)$ \\
\hline \multicolumn{2}{|l|}{ Maternal ethnicity } \\
\hline Caucasian & $9,243(96.4 \%)$ \\
\hline Asian & $110(1.1 \%)$ \\
\hline African & $232(2.4 \%)$ \\
\hline \multicolumn{2}{|l|}{ Maternal smoking habits } \\
\hline Yes & $1,546(16.1 \%)$ \\
\hline No & $8,039(83.9 \%)$ \\
\hline \multicolumn{2}{|l|}{ Ultrasound parameters at 35 (34-36) weeks } \\
\hline Gestational age (weeks) at ultrasound & $35.1(35.0-35.3)$ \\
\hline EFW, g by Hadlock & $2,495(2,314-2,697)$ \\
\hline EFW, g by Stirnemann & $2,421(2,209-2,648)$ \\
\hline Percentile by standard & P50 (P10-P90) \\
\hline NC MSUH & $52.6(11.9-93.3)$ \\
\hline Customized MSUH & $52.9(12.2-92.9)$ \\
\hline Figueras & $59.3(18.1-93.5)$ \\
\hline INTERGROWTH-21st & $51.9(12.7-89.8)$ \\
\hline WHO & $43.1(7.5-74.9)$ \\
\hline FMF & $37.6(2.7-89.9)$ \\
\hline \multicolumn{2}{|l|}{ Pregnancy and perinatal outcomes } \\
\hline Gestational age at delivery, weeks & $40.0(39.1-40.7)$ \\
\hline \multicolumn{2}{|l|}{ Newborn gender } \\
\hline Female & $4,652(48.5 \%)$ \\
\hline Male & $4,933(51.5 \%)$ \\
\hline Birth weight, g & $3,310(3,030-3,590)$ \\
\hline LGA & $961(10.02 \%)$ \\
\hline
\end{tabular}

SSD-5000 (Hitachi Aloka Medical Systems, Tokyo, Japan) ultrasound machines. EFW was calculated with the formula of each model developed using the formula of Hadlock et al. [7] with biparietal diameter, cephalic and abdominal circumference, and femur length for the MSHU, Figueras et al. [16], WHO standards; cephalic and abdominal circumference, and femur length for the Fetal Medicine Foundation (FMF) standard. In addition, for the same reason, Stirnemann et al.'s [12] formula, which considers only cephalic and abdominal circumference, was used to estimate percentile weight for INTERGROWTH-21st.

The variables included in the study were maternal age and BMI at the beginning of pregnancy, parity, maternal and paternal height, maternal ethnic origin, smoking habits, infant gender, birth weight, and ultrasound EFW. We also collected perinatal outcomes to analyze APOs in LGAs at delivery, defined as the oc- currence of 5-min Apgar score $<7$, instrumental or cesarean delivery for non-reassuring fetal status (NRFS), arterial cord blood $\mathrm{pH}$ $<7.10$, and stillbirth.

\section{Estimated Percentile Weight}

EPWs were calculated according to 6 different customized and NC growth standards including population, population-customized, and international references. For the customized charts, Hadlock et al.'s [6] and Gardosi et al.'s [8] methodologies were used to develop (1) the MSUH standard customized for parity, age, BMI and maternal height, paternal height, and fetal gender, built using a modified version of Hadlock et al. [6,7] growth charts adjusted to our population with a coefficient of variation that changes with gestational age (Savirón-Cornudella et al. [17]); (2) and the Barcelona Clinic Hospital (Figueras et al. [16]). For the NC standards, 
Table 2. Diagnosis of APOs

\begin{tabular}{|c|c|c|c|c|c|c|}
\hline & $\begin{array}{l}\text { 5-min Apgar } \\
\text { score }<7\end{array}$ & $\begin{array}{l}\text { Instrumental } \\
\text { delivery for NRFS }\end{array}$ & $\begin{array}{l}\text { Cesarean } \\
\text { delivery for NRFS }\end{array}$ & $\begin{array}{l}\text { Arterial cord } \\
\text { blood } \mathrm{pH}<7.10\end{array}$ & Stillbirth & Any APO \\
\hline Total cohort & 42 & 161 & 265 & 254 & 19 & 645 \\
\hline LGA, $n(\%)$ & $1(2.4)$ & $13(8.1)$ & $17(6.4)$ & $16(6.3)$ & $1(5.3)$ & $44(9.5)$ \\
\hline \multicolumn{7}{|l|}{$E P W>90, \mathrm{n}(\%)$} \\
\hline NC MSUH & $4(9.5)$ & $11(6.8)$ & $33(12.5)$ & $25(9.8)$ & $3(15.8)$ & $67(10.4)$ \\
\hline Customized MSUH & $4(9.5)$ & $16(9.9)$ & $37(14.0)$ & $30(11.8)$ & $3(15.8)$ & $79(12.2)$ \\
\hline Figueras & $5(11.9)$ & $16(9.9)$ & $36(13.6)$ & $34(13.4)$ & $4(21.1)$ & $81(12.6)$ \\
\hline INTERGROWTH-21st & $3(7.1)$ & $8(5.0)$ & $25(9.4)$ & $17(6.7)$ & $2(10.5)$ & $50(7.8)$ \\
\hline $\mathrm{WHO}$ & $0(0)$ & $6(3.7)$ & $6(2.3)$ & $4(1.6)$ & $0(0)$ & $16(2.5)$ \\
\hline FMF & $3(7.1)$ & $9(5.6)$ & $27(10.2)$ & $17(6.7)$ & $2(10.5)$ & $52(8.1)$ \\
\hline
\end{tabular}

APOs, adverse perinatal outcomes; NC, non-customized; MSUH, Miguel Servet University Hospital; FMF, Fetal Medicine Foundation; NRFS, non-reassuring fetal status.

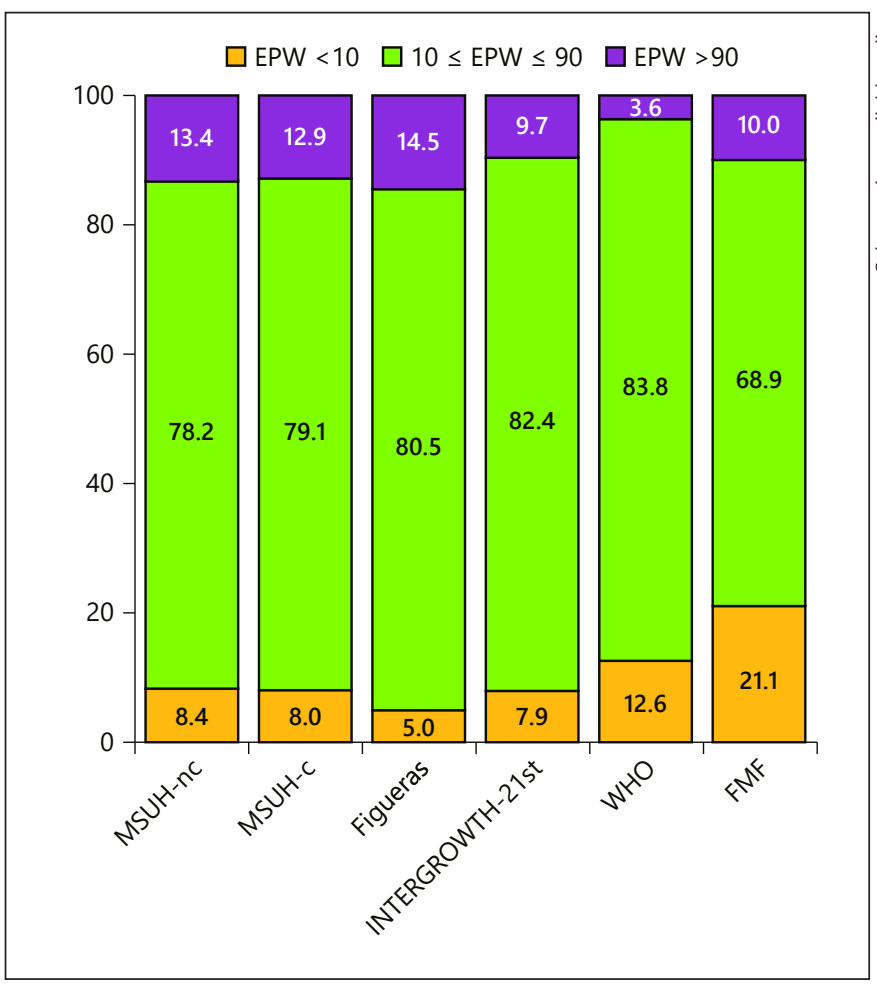

Fig. 2. Bar diagram of percentages of small for gestational age (Orange), adequate for gestational age (Green), and LGA (Purple) provided by standards at 3rd trimester ultrasound (range 34-36 weeks). FMF, Fetal Medicine Foundation; MSUH, Miguel Servet University Hospital; WHO, World Health Organization; LGA, large for gestational age; EPW, estimated percentile weight.

we used (iii) a NC version of the MSUH standard (Savirón-Cornudella et al. [17]); (4) the international population INTERGROWTH-21st $[12,13]$, a multilevel mixed model which includes pregnant women without any pathology; (5) the international
WHO fetal growth standard [14]; and (6) the FMF local growth multilevel mixed model (Nicolaides et al. [18]).

To assess ultrasound weight measures in the 3rd trimester, EPWs were estimated between 34 and 36 weeks of gestational age. The WHO EPW was calculated by interpolation of 5, 10, 25, 50, 75, 90 , and 95 percentiles. As a gold standard for the analysis, LGA was defined as percentile birth weight over 90 , using a growth reference for the Spanish population based on 9,362 birth weights [19].

\section{Statistical Analysis}

Once the data had been extracted, a descriptive table with the variables by ultrasound and at delivery was created. The predictive ability of EPW provided by the 6 standards used to predict LGAs was analyzed using the area under the receiver-operating characteristic curve (AUC) [20]. Sensitivity (detection rate) was established for false-positive rates (FPRs) of 5, 10, 15, and 20\%. The percentile threshold point corresponding to the FPR values was also calculated. AUCs were compared using the DeLong test and sensitivities through a proportion comparison test. In addition, a sub-analysis was performed using a logistic regression model to estimate the odds ratio (OR) and $95 \%$ confidence interval (CI) for an increase in $1 \%$ of the EPW at 35 weeks as a predictor for LGAs at delivery, performing a subclassification for different ultrasounddelivery intervals (1-6 weeks).

We analyzed the diagnostic ability of the standards using EPW $>90$, and LGA birth weights to detect APOs: 5-min Apgar score $<7$, instrumental delivery for NRFS, cesarean delivery for NRFS, arterial cord blood $\mathrm{pH}<7.10$, and stillbirth. Comparison between APOs predicted by standards was performed using a proportion test. The analyses were performed using $\mathrm{R}$ version 3.6.1 language programming ( $\mathrm{R}$ Foundation for Statistical Computing, Vienna, Austria) [21].

\section{Results}

Descriptive Results

Table 1 shows the descriptive characteristics of the subjects and displays medians and interquartile ranges 
Table 3. Area under the receiver-operating characteristic curve and sensitivity analyses to detect LGA newborns, using EPW by ultrasound at 35 weeks (range $34-36$ weeks) for different FPR percentages

\begin{tabular}{|c|c|c|c|c|c|}
\hline \multirow[t]{2}{*}{ Standard } & \multirow[t]{2}{*}{$\operatorname{AUC}(95 \% \mathrm{CI})$} & \multicolumn{4}{|c|}{ Sensitivity $\left(95 \%\right.$ CI) and threshold percentile points ${ }^{\mathrm{a}}$} \\
\hline & & FPR 5\% & FPR $10 \%$ & FPR $15 \%$ & FPR $20 \%$ \\
\hline NC MSUH & $0.83(0.82-0.85)$ & $42.5(39.3-45.7)$ (Thr 94.2) & $55.8(52.6-58.9)(\mathrm{Thr} 88.6)$ & $66.8(63.7-69.8)(\mathrm{Thr} 83.6)$ & $73.8(70.8-76.5)($ Thr 78.3$)$ \\
\hline Customized MSUH & $0.80(0.78-0.81)$ & $30.0(27.1-33.0)($ Thr 95.0$)$ & $45.3(42.1 .48 .5)(\mathrm{Thr} 89.5)$ & $55.2(51.9-58.3)($ Thr 84.3$)$ & $62.7(59.6-65.8)($ Thr 79.2$)$ \\
\hline Figueras & $0.81(0.79-0.82)$ & $32.3(29.3-35.3)($ Thr 95.4$)$ & $48.4(45.2-51.6)($ Thr 90.5) & $58.4(55.2-61.5)($ Thr 86.1$)$ & $64.4(61.3-67.4)($ Thr 81.8$)$ \\
\hline INTERGROWTH-21st & $0.84(0.82-0.85)$ & $36.3(33.3-39.5)($ Thr 91.5) & $51.0(47.8-54.2)($ Thr 85.9$)$ & $62.0(58.9-65.1)(\mathrm{Thr} 80.1)$ & $72.1(69.1-74.9)($ Thr 75.3$)$ \\
\hline WHO & $0.77(0.76-0.79)$ & $25.9(23.2-28.8)($ Thr 81.9) & $31.2(28.3-34.3)(\mathrm{Thr} 74.5)$ & $35.3(32.3-38.4)($ Thr 73.7) & $41.7(38.6-44.9)($ Thr 72.5$)$ \\
\hline FMF & $0.85(0.84-0-86)$ & $38.2(35.1-41.4)$ (Thr 92.2) & $56.5(53.3-59.7)(\mathrm{Thr} 84.2)$ & $65.7(62.5-68.6)($ Thr 76.9) & $72.8(69.9-75.6)($ Thr 69.8) \\
\hline
\end{tabular}

MSUH, Miguel Servet University Hospital; Pc, percentile; WHO, World Health Organization; LGA, large for gestational age; NC, non-customized; FMF, Fetal Medicine Foundation; AUC, area under the curve; FPR, false-positive rate; CI, confidence interval; EPW, estimated percentile weight. ${ }^{\text {a }}$ Sensitive threshold percentile: point that corresponds to a FPR value.

Table 4. Results of $p$ value tests to compare standards: AUC and sensitivities (specificity 90\%) to predict LGA, and percentage of APO diagnosis

\begin{tabular}{|c|c|c|c|c|c|c|c|c|c|c|c|c|c|c|c|}
\hline & \multicolumn{3}{|c|}{ Customized MSUH } & \multicolumn{3}{|c|}{ Figueras } & \multicolumn{3}{|c|}{ INTERGROWTH-21st } & \multicolumn{3}{|l|}{ WHO } & \multicolumn{3}{|l|}{ FMF } \\
\hline Customized MSUH & & & & 0.002 & 0.188 & 0.933 & $<0.001$ & 0.014 & 0.009 & $<0.001$ & $<0.001$ & $<0.001$ & $<0.001$ & $<0.001$ & 0.017 \\
\hline Figueras & & & & & & & $<0.001$ & 0.274 & 0.006 & $<0.001$ & $<0.001$ & $<0.001$ & $<0.001$ & $<0.001$ & 0.010 \\
\hline INTERGROWTH-21st & & & & & & & & & & $<0.001$ & $<0.001$ & $<0.001$ & $<0.001$ & 0.018 & 0.918 \\
\hline WHO & & & & & & & & & & & & & $<0.001$ & $<0.001$ & $<0.001$ \\
\hline
\end{tabular}

NC, non-customized; MSUH, Miguel Servet University Hospital; FMF, Fetal Medicine Foundation; WHO, World Health Organization; APO, adverse perinatal outcome; AUC, area under the curve.

among groups for the 6 studied standards for EFWs by ultrasound at 35 weeks (range from $34+0$ to $36+6$ weeks) and weights at delivery and EPWs. Both WHO and FMF standards show an underestimation of the expected value by ultrasound (median values $43.1 \%$, interquartile range $7.5-74.9$, and $37.6 \%$, interquartile range $2.7-89.9$, respectively), and similarly, the Figueras standard shows an overestimation by ultrasound (median values $59.3 \%$, interquartile range 18.1-93.5). EPW distributions are detailed in Figure 2, where a comparison of bar diagrams is shown for each standard. The rate of LGA at birth was $10.0 \%(n=961)$. Regarding APOs, Table 2 shows that LGA deliveries include 9.5\% $(n=44)$ APOs, $2.4 \%(n=1)$ 5-min Apgar score $<7,8.1 \%(n=13)$ instrumental deliveries for NRFS, $6.4 \%(n=17)$ cesarean deliveries for NRFS, 6.3\% $(n=16)$ neonatal acidemia (pH cord blood $\mathrm{pH}<7.10)$, and $5.3 \%(n=1)$ stillbirth.

\section{Comparison of Growth Standards}

Table 3 displays values of AUCs and sensitivities plus the percentile threshold point for different FPRs to predict LGAs at delivery by ultrasound. For a $10 \%$ FPR, the detection rates for LGAs for all the standards ranged be-

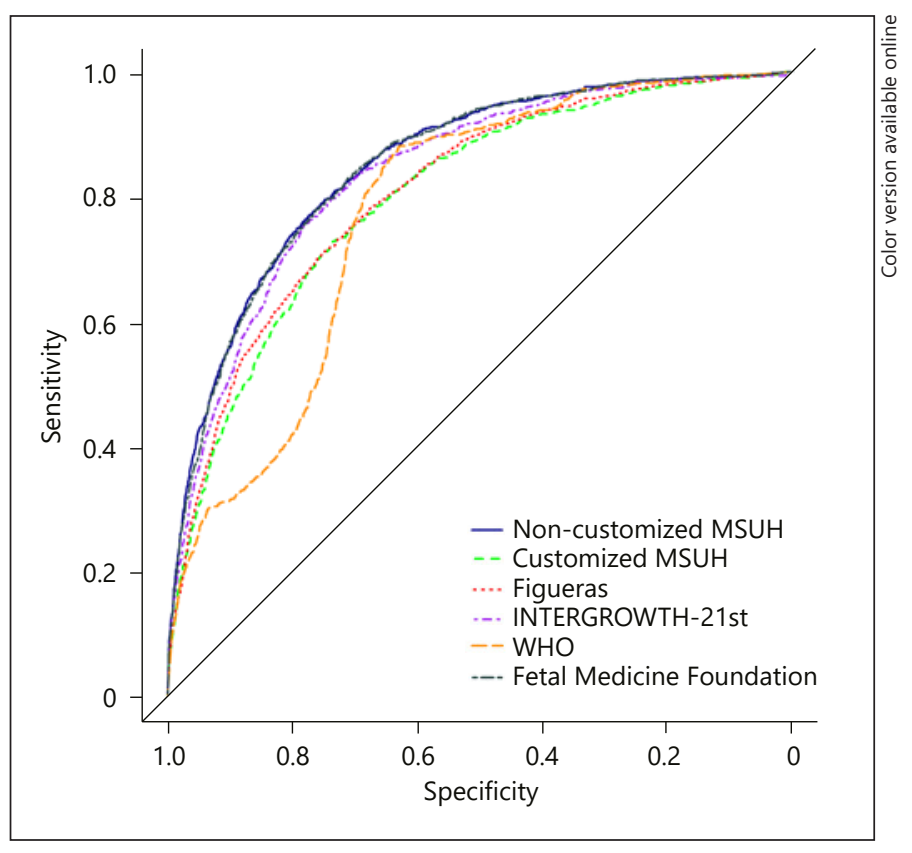

Fig. 3. Receiver-operating characteristic curves: comparison of fetal growth standards for prediction of LGA newborns using EPW by ultrasound at 35 weeks. MSUH, Miguel Servet University Hospital; WHO, World Health Organization. 
Fig. 4. ORs and 95\% CIs of standards in order to predict LGA newborns according to EPW by ultrasound at 35 weeks. MSUH, Miguel Servet University Hospital; WHO, World Health Organization; ORs, odds ratios; CIs, confidence intervals.

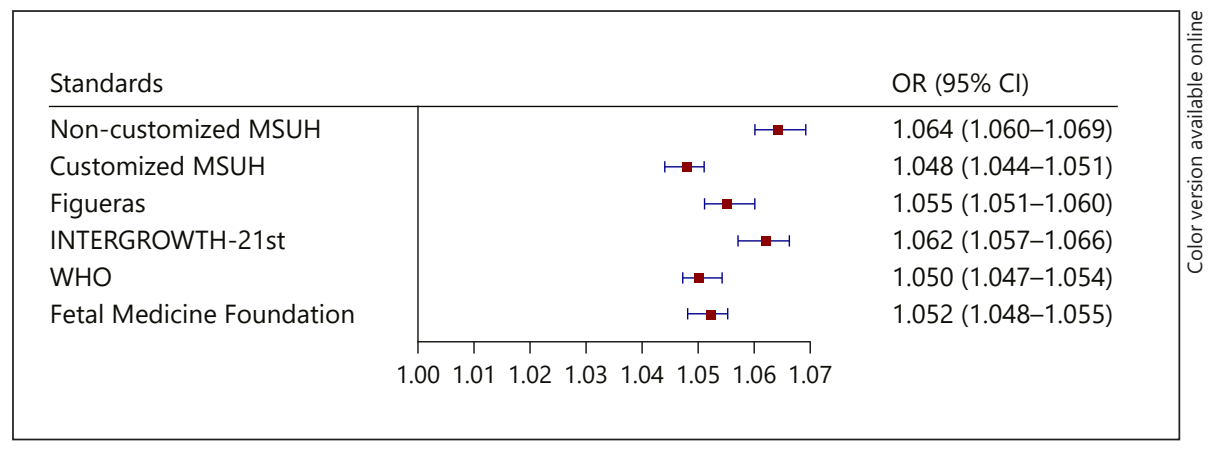

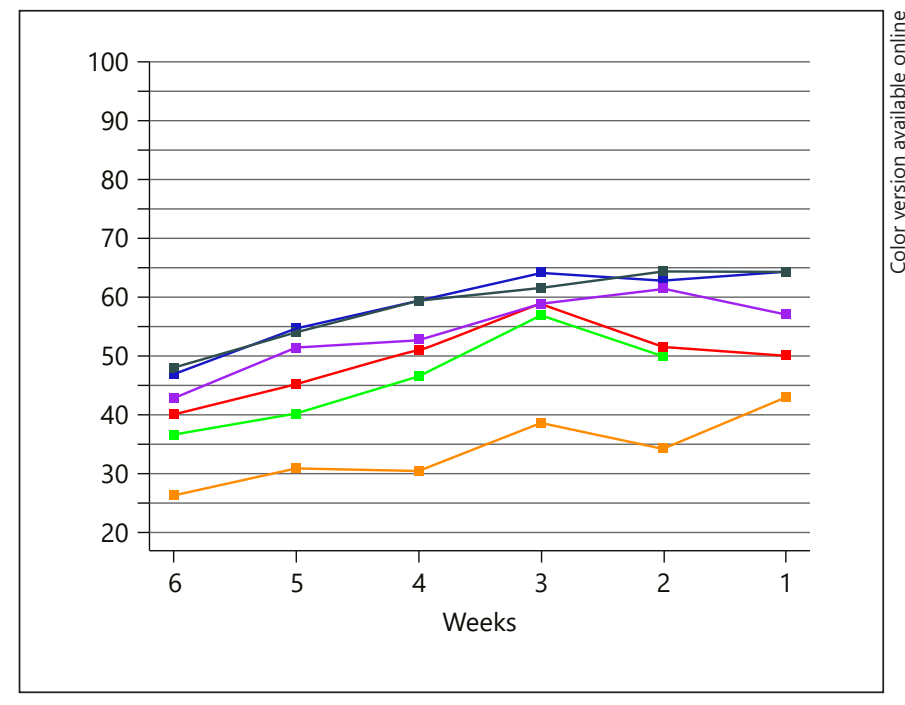

Fig. 5. Prediction of LGA cases by standards by ultrasound-delivery interval (1-6 weeks) for a 10\% FPR. Growth standards: NC MSUH [17] (dark blue line), customized MSUH [17] (green line), Figueras et al. [16] (red line), INTERGROWTH-21st [13] (purple line), WHO [14] (orange line), and FMF [18] (dark slate grey line). LGA, large for gestational age; NC, non-customized; MSUH, Miguel Servet University Hospital; WHO, World Health Organization; FMF, Fetal Medicine Foundation; FPR, false-positive rate.

tween $31.2 \%$ with the WHO standard (AUC $0.77 ; 95 \%$ CI, $0.76-0,79$ ) and $56.5 \%$ with the FMF standard (AUC 0.85; $95 \%$ CI, 0.84-0.86). These values were obtained with percentile threshold points over 88.6, 89.5, 90.5, 85.9, 74.5, and $84.2 \%$ for, respectively, NC and customized MSUH, Figueras, INTERGROWTH-21st, WHO, and FMF standards. For a $20 \%$ FPR, the detection rates ranged from 41.7 to $73.8 \%$, using $78.3,79.2,81.8,75.3,72.5 \%$, and $69.8 \%$ as the percentile threshold points.

Figure 3 illustrates the receiver-operating characteristic curve comparison and the AUC for the prediction of
LGAs at delivery by ultrasound at 35 weeks. Figure 4 displays the results of the logistic regression model with the ORs and 95\% CIs to predict LGAs by ultrasound at 35 weeks according to the standards. Online suppl. Table 1 (for all online suppl. material, see www.karger.com/ doi/10.1159/000510020) shows the concordance between predicted LGAs at delivery by standards using EPW $>90$.

$p$ Values of the comparisons of the standard AUC values and sensitivity for a 90\% specificity are shown in Table 4 . The FMF and the NC MSUH standards showed no statistically significant differences between them, but with greater LGA prediction ability than INTERGROWTH-21st, Figueras, and WHO standards. Besides, in the comparison, INTERGROWTH-21st showed significant differences from customized MSHU and WHO standards, but not with the Figueras standard. Finally, customized standards did not show differences between them, but with greater LGA prediction ability than the WHO standard.

Regarding APO prediction by EPW $>90$, in Table 2 we show that the customized Figueras and the MSUH standards reached the greatest detection rate, 12.6 and $12.2 \%$, respectively. No statistically significant difference was detected with the NC MSUH (10.4\%), although the differences with the FMF (8.1\%), INTERGROWTH-21st (7.8\%), and WHO (2.5\%) standards were significant. The instrumental deliveries for NRFS, cesarean deliveries for NRFS, and neonatal acidemia APOs might explain those differences. $p$ values of all comparisons are illustrated in Table 4.

\section{Comparison of Growth Standards by Ultrasound- \\ Delivery Interval}

Online suppl. Table 2 displays values of AUCs and sensitivities for different FPRs to predict LGAs by ultrasound-delivery interval (range 1-6 weeks). Figure 5 shows the prediction of LGA cases by standard by ultrasounddelivery interval (1-6 weeks) for a $10 \%$ FPR. The ob- 
served results show higher detection rates as the interval decreases. Online suppl. Figure 1 illustrates the receiveroperating characteristic curve comparison of fetal growth standards for the prediction of LGA newborns according to the ultrasound-delivery interval (range 1-6 weeks). Online suppl. Figure 1 displays ORs and 95\% CIs of standards in order to predict LGAs by ultrasound-delivery interval (range 1-6 weeks).

\section{Discussion}

\section{Principal Findings}

Our study has demonstrated the utility of EPW by ultrasound exam at 35 weeks (range $34+0$ to $36+6$ weeks) as a predictor of LGAs at delivery at term with any model studied. The comparison of 6 growth standards showed similar good predictive ability for LGAs, and with the exception of the WHO standard, NC standards present a greater predictive ability to detect LGAs at delivery. The NC MSHU and FMF standards fit better to a $10 \%$ detection of LGAs at delivery and with an ultrasound-delivery interval of 1 week; therefore, MSHU and FMF standards have a higher detection rate. On the contrary, when we analyzed LGAs with the APO predictive ability of the 6 standards by percentile weight $>90$ at the 35th week of gestational age, customized Figueras and MSUH standards showed the greatest diagnostic ability, with statistically significant differences with 3 of the 4 NC models. A previous study did not find any significant difference between customized and NC standards analyzing the predictive ability of EPW to detect APOs; by contrast, using EPW $>90$, we detected significant differences [9]. The shorter ultrasound-delivery interval is related to better prediction rates with all the standards.

The predictive capacity for LGAs at delivery was not perfect for the 6 growth standards however, and several studies of ultrasound accuracy have consistently reported an EFW underestimation in LGAs due to the intrinsic error of ultrasound [22], which could, at least partially, justify these detection rates. Lowering the percentile threshold point by ultrasound would, in our view, improve the detection of LGAs at delivery but would increase FPRs. In any case, unless the birth is scheduled, it will be difficult to identify the best time to perform the ultrasound.

\section{Prediction of LGAs at Delivery by Ultrasound}

Sovio et al. [5] in 2018 supported universal 36-week ultrasound versus selective scans to screen for LGA in- fants, with an improvement in detection rates from $27 \%$ (FPR $1.7 \%$ and AUC 0.71 ) to $38 \%$ (FPR $3.2 \%$ and AUC 0.87 ). Their study included preterm deliveries and a low rate of LGAs of $4.6 \%$, which could explain the lower FPR in their study.

In 2012, Zhang et al. [23] showed a higher predictive ability for LGA infants when an ultrasound was performed close to delivery (AUC 0.843 at 33 weeks and AUC 0.889 at 37 weeks). Souka et al.'s [24] 2013 study reported a $53 \%$ detection rate (FPR $10 \%$ and AUC 0.85) by ultrasound at 32-33 weeks, which increased to $63 \%$ (FPR 10\% and AUC 0.87) by ultrasound at 35-36 weeks. Their study matched our results, although it is based on different inclusion criteria (LGA infants defined as higher than the 95 percentile). This is probably because the fetus has less time to modify its weight percentile until birth as the ultrasound-delivery interval decreases.

In a similar line, a longitudinal cohort study by Tarca et al. [25], carried out in 2016, displayed a detection rate of $54 \%$ of LGA infants (FPR $10 \%$ and AUC 0.84 ). A possible reason for their lower detection rates might be associated to the earlier ultrasound at 34 weeks. Frick et al. [26] also published similar results scanning at 30-34 weeks with a $41.3 \%$ detection rate (FPR $10 \%$ and AUC 0.760 ), which increased to $48.6 \%$ at $35-37$ weeks (FPR $10 \%$ and AUC 0.803). Their work considered maternal factors and also emphasized that the ability to predict increases in LGA infants when estimation of weight assessment was performed closer to delivery.

We could argue that the cutoff point of the 90th percentile by ultrasound seems to be limited to the prediction of LGAs at delivery. Screening by maternal factors and biomarkers in the 3 trimesters of pregnancy has been developed for LGA neonates similar to SGA standards, but with limited results [25]. Understandably, further studies should be conducted which can improve the detection rate of LGAs.

\section{Strengths and Limitations of the Study}

As one of the strengths of our research, we should highlight the large sample size of the data analyzed, approximately 10,000 pregnancies. It is also noteworthy that our statistical approach and comparison present a low risk of bias. However, among the limitations of our research, we should mention the fact that our study is restricted to 1 population with a high proportion of Caucasians (96.4\%), and our results might therefore show a risk of bias due to the retrospective nature of the study. Hence, future studies might benefit from a more detailed prospective protocol. 


\section{Clinical Relevance}

The predictive ability of ultrasound EFW at 35 weeks for LGA infants is similarly good for the 6 growth standards, despite the significant improvement detected in the use of NC local or international standards with no statistically significant differences between them. In contrast, when focusing on the use of EPW $>90$ at week 35 for the prediction of LGAs with APOs, customized standards demonstrated an advantage over NC standards. We would thus conclude that standards with a shorter ultrasound-delivery interval would be related to better prediction rates.

\section{Acknowledgements}

We gratefully thank all the staff of the Ultrasound and Prenatal Diagnosis Unit of the MSUH (Zaragoza, Spain) for their help in the compilation and management of the data, and Concepción Orna for her help in language editing.

\section{Statement of Ethics}

The research presented in the manuscript was conducted ethically in accordance with the World Medical Association Declaration of Helsinki and the appropriate guidelines for human studies, and was approved by the research institute's committee on human research (Clinical Research Ethics Committee of Aragon PI20/ 414). No patient consents were required.

\section{Conflict of Interest Statement}

The authors have no conflicts of interest to declare.

\section{Funding Sources}

The study has no financial support.

\section{Author Contributions}

R.S.C., L.M.E., and R.A.G. contributed to the conception of the study. R.S.C., L.M.E., F.R.P.L., B.C.L., and M.T.D. contributed to the design of the work. P.D.P. and M.T.D. carried out data acquisition. L.M.E., R.A.G., and G.S. performed statistical analyses. All authors were involved in the interpretation of the study results, and the drafting and revision of the manuscript, and all approved the final version to be published.

\section{References}

1 Vasak B, Koenen SV, Koster MP, Hukkelhoven CW, Franx A, Hanson MA, et al. Human fetal growth is constrained below optimal for perinatal survival. Ultrasound Obstet Gynecol. 2015 Feb;45(2):162-7.

2 Carter EB, Stockburger J, Tuuli MG, Macones GA, Odibo AO, Trudell AS. Large-for-gestational age and stillbirth: is there a role for antenatal testing? Ultrasound Obstet Gynecol. 2019 Sep;54(3):334-7.

3 Salomon LJ, Alfirevic Z, Da Silva Costa F, Deter RL, Figueras F, Ghi T, et al. ISUOG practice guidelines: ultrasound assessment of fetal biometry and growth. Ultrasound Obstet Gynecol. 2019 Jun;53(6):715-23.

4 Boulvain M, Senat MV, Perrotin F, Winer N, Beucher G, Subtil D, et al. Induction of labour versus expectant management for large-fordate fetuses: a randomised controlled trial. Lancet. 2015;385(9987):2600-5.

5 Sovio U, Moraitis AA, Wong HS, Smith GCS. Universal versus selective ultrasonography to screen for large for gestational age infants and associated morbidity. Ultrasound Obstet Gynecol. 2018 Jun;51(6):783-91.

6 Hadlock FP, Harrist RB, Martinez-Poyer J. In utero analysis of fetal growth: a sonographic weight standard. Radiology. 1991 Oct;181(1): 129-33.
7 Hadlock FP, Harrist RB, Sharman RS, Deter RL, Park SK. Estimation of fetal weight with the use of head, body, and femur measurements: a prospective study. Am J Obstet Gynecol. 1985;151(3):333-7.

8 Gardosi J, Chang A, Kalyan B, Sahota D, Symonds EM. Customised antenatal growth charts. Lancet. 1992;339(8788):283-7.

9 Savirón-Cornudella R, Esteban LM, TajadaDuaso M, Castán-Mateo S, Dieste-Pérez P, Cotaina-Gracia L, et al. Detection of adverse perinatal outcomes at term delivery using ultrasound estimated percentile weight at 35 weeks of gestation: comparison of five fetal growth standards. Fetal Diagn Ther. 2020; 47(2):104-14.

10 Kabiri D, Romero R, Gudicha DW, Hernandez-Andrade E, Pacora P, Benshalom-Tirosh $\mathrm{N}$, et al. Prediction of adverse perinatal outcome by fetal biometry: comparison of customized and population-based standards. Ultrasound Obstet Gynecol. 2020 Feb;55(2): $177-88$.

11 Ohuma EO, Altman DG. Statistical methodology for constructing gestational age-related charts using cross-sectional and longitudinal data: the INTERGROWTH-21st Project as a case study. Stat Med. 2019 Aug 30;38(19): 3507-26.
12 Stirnemann J, Villar J, Salomon LJ, Ohuma E, Ruyan P, Altman DG, et al. International estimated fetal weight standards of the INTERGROWTH-21st project. Ultrasound Obstet Gynecol. 2017 Apr;49(4):478-86.

13 Villar J, Cheikh Ismail L, Victora CG, Ohuma EO, Bertino E, Altman DG, et al. International standards for newborn weight, length, and head circumference by gestational age and sex: the newborn cross-sectional study of the INTERGROWTH-21st project. Lancet. 2014; 384(9946):857-68.

14 Kiserud T, Piaggio G, Carroli G, Widmer M, Carvalho J, Neerup Jensen L, et al. The world health organization fetal growth charts: a multinational longitudinal study of ultrasound biometric measurements and estimated fetal weight. PLoS Med. 2017;14(1): e1002220.

15 Committee on Obstetric Practice, the American Institute of Ultrasound in Medicine, and the Society for Maternal-Fetal Medicine. Committee Opinion No 700: methods for estimating the due date. Obstet Gynecol. 2017 May;129(5):e150-4.

16 Figueras F, Meler E, Iraola A, Eixarch E, Coll O, Figueras J, et al. Customized birthweight standards for a spanish population. Eur J Obstet Gynecol Reprod Biol. 2008 Jan;136(1):20-4. 
17 Savirón-Cornudella R, Esteban LM, Lerma D, Cotaina L, Borque Á, Sanz G, et al. Comparison of fetal weight distribution improved by paternal height by Spanish standard versus INTERGROWTH 21st standard. J Perinat Med. 2018 Sep 25;46(7):750-9.

18 Nicolaides KH, Wright D, Syngelaki A, Wright A, Akolekar R. Fetal medicine foundation fetal and neonatal population weight charts. Ultrasound Obstet Gynecol. 2018 Jul; 52(1):44-51.

19 Carrascosa A, Fernández JM, Ferrández A, López-Siguero JP, López D, Sánchez E, et al. Estudios españoles de crecimiento 2010. Rev Esp Endocrinol Pediatr. 2011;2(1):59-62.

20 Hanley JA, McNeil BJ. The meaning and use of the area under a receiver operating characteristic (ROC) curve. Radiology. 1982 Apr; 143(1):29-36
21 R Core Team. R: a language and environment for statistical computing. Vienna, Austria: R Foundation for Statistical Computing; 2014.

22 Mongelli M, Benzie R. Ultrasound diagnosis of fetal macrosomia: a comparison of weight prediction models using computer simulation. Ultrasound Obstet Gynecol. 2005 Oct; 26(5):500-3.

23 Zhang J, Kim S, Grewal J, Albert PS. Predicting large fetuses at birth: do multiple ultrasound examinations and longitudinal statistical modelling improve prediction? Paediatr Perinat Epidemiol. 2012 May;26(3):199-207.
24 Souka AP, Papastefanou I, Pilalis A, Michalitsi V, Panagopoulos P, Kassanos D. Performance of the ultrasound examination in the early and late third trimester for the prediction of birth weight deviations. Prenat Diagn. 2013;33(10):915-20.

25 Tarca AL, Hernandez-Andrade E, Ahn H, Garcia M, Xu Z, Korzeniewski SJ, et al. Single and serial fetal biometry to detect preterm and term small- and large-for-gestational-age neonates: a longitudinal cohort study. PLoS One. 2016 Nov 1;11(11):e0164161.

26 Frick AP, Syngelaki A, Zheng M, Poon LC, Nicolaides KH. Prediction of large-for-gestational-age neonates: screening by maternal factors and biomarkers in the three trimesters of pregnancy. Ultrasound Obstet Gynecol. 2016 Mar;47(3):332-9. 